002

\title{
HEPARIN-BINDING EGF-LIKE GROWTH FACTOR (HB-EGF): CAN WE ADD THIS TO BREAST MILK IN THE FUTURE TO PREVENT NECROTIZING ENTEROCOLITIS
}

\author{
G.E. Besner, J. Yang, D. Watkins, Y. Zhou, C.-L. Chen \\ Department of Pediatric Surgery, Nationwide Children's Hospital, Columbus, OH, USA
}

\begin{abstract}
We have previously shown that heparin-binding EGF-like growth factor (HB-EGF), a glycoprotein that is naturally present in low levels in human breast milk, protects the intestines from injury in several different animal models, including a model of neonatal necrotizing enterocolitis (NEC). In addition to its trophic effects which are mediated by its ability to stimulate intestinal epithelial cell proliferation and migration, HB-EGF prevents the production of injurious mediators of intestinal injury, and acts as an anti-inflammatory factor. More recently, we have shown that HB-EGF protects intestinal stem cells (ISC) from injury in our animal model of NEC. We believe that the ability of HB-EGF to protect the intestines from NEC may be due, in large part, to its effects on ISC. Ongoing studies in our lab have examined the ability of HB-EGF to act synergistically with transplanted mesenchymal stem cells (MSC) in protection of the intestines from NEC. HB-EGF stimulates MSC proliferation and migration in vitro, and promotes transplanted MSC engraftment into injured intestine in vivo. The administration of both HB-EGF and MSC decrease the incidence and severity of NEC, and increased survival, in our model. In addition, we have recently shown that HB-EGF protects the enteric nervous system from injury, and acts to promote intestinal motility. Collectively, these findings lend further support for the use of HB-EGF in the future as a potential therapy for the prevention or treatment of NEC.
\end{abstract}

